\title{
Interference with NTSR1 Expression Exerts an Anti-Invasion Effect via the Jun/miR- 494/SOCS6 Axis of Glioblastoma Cells
}

\author{
Qing Ou-yang Xuzhi He Anqi Yang Bing Li Minhui Xu \\ Department of Neurosurgery, Daping Hospital, Army Medical University, Chongqing, China
}

\author{
Key Words \\ Glioblastoma $・$ Invasion $・$ MicroRNA $・$ NTSR1 $・$ SOCS6
}

\begin{abstract}
Background/Aims: Glioblastoma is the most common and aggressive brain tumor and carries a poor prognosis. Previously, we found that neurotensin receptor 1 (NTSR1) contributes to glioma progression, but the underlying mechanisms of NTSR1 in glioblastoma invasion remain to be clarified. The aim of this study was to investigate the molecular mechanisms of NTSR1 in glioblastoma invasion. Methods: Cell migration and invasion were evaluated using woundhealing and transwell assays. Cell proliferation was detected using CCK-8. The expression of NTSR1, Jun, and suppressor of cytokine signaling 6 (SOCS6) was detected using western blotting. The expression of miR-494 was detected by Quantitative real-time PCR. Chromatin immunoprecipitation assay was performed to examine the interaction between Jun and miR494 promoter. Dual-luciferase reporter assay and western blotting were performed to identify the direct regulation of SOCS6 by miR-494. An orthotopic xenograft mouse model was conducted to assess tumor growth and invasion. Results: NTSR1 knockdown attenuated the invasion of glioblastoma cells. Jun was positively regulated by NTSR1, which promoted miR494 expression through binding to miR-494 promoter. SOCS6 was confirmed as a direct target of miR-494, thus, NTSR1-induced miR-494 upregulation resulted in SOCS6 downregulation. Both miR-494 and SOCS6 were involved in the NTSR1-induced invasion of glioblastoma cells. In vivo, tumor invasion and growth were inhibited by NTSR1 knockdown, but were restored with miR-494 overexpression. Conclusion: NTSR1 knockdown inhibited glioblastoma invasion via the Jun/miR-494/SOCS6 axis.
\end{abstract}




\section{Introduction}

Glioblastoma is the most common and lethal malignant tumor of the central nervous system [1]. In glioblastoma multiforme (GBM), patients are treated with surgical resection, radiation therapy, chemotherapy and targeted therapy, but the median overall survival of GBM patients remains 12-15 months [2-6]. A highly aggressive nature is one of the hallmarks of glioblastoma, and is closely related to the malignant progression of glioblastoma and poor prognosis of patients. Local invasion and growth of glioblastoma complicates complete surgical resection, and the inevitable remnant tumor mass predictably leads to easy recurrence and high mortality after surgery; therefore, radical resection of glioblastoma is not curative $[7,8]$. In addition to migratory activity, proteolytic degradation of the extracellular matrix (ECM) is a critical element of cancer invasion. In general, cancer cells produce matrix metalloproteinases to degrade ECM components. Moreover, tumor invasion is also controlled by the local microenvironment including autocrine and paracrine factors, such as epidermal growth factor and transforming growth factor beta.

NTSR1 stimulation triggers the activation of various intracellular signaling pathways, including extracellular signal-regulated kinase 1/2 (ERK1/2), Rho GTPases, and focal adhesion kinase, leading to gene transcriptional activation and tumor growth $[9,10]$. NTSR1 is commonly overexpressed in glioma and expression levels of NTSR1 are found to correlate positively with pathological grade, but negatively with prognosis in glioma patients [11]. Several reports indicate that once activated by ligands, NTSR1 signaling is involved in a variety of processes such as cell proliferation, apoptosis, and stemness maintenance of glioma cells; these effects can be abolished by SR48692 (a specific antagonist of NTSR1) or by NTSR1 knockdown [12-14]. In a previous report, we described how NTSR1 knockdown inhibits invasion of glioma cells, however, the molecular mechanisms underlying NTSR1mediated glioblastoma invasion need further investigation.

MicroRNAs (miRNAs) are a class of short, single-stranded non-coding RNA, which bind to targets with near perfect complementarity to bring about translational repression or transcript degradation of targets [15]. Accumulating evidence has shown that miRNAs participate in the progression of cancers, and have been categorized either as oncogenic miRNAs and tumor suppressor miRNAs depending upon their function [16]. Especially, it has been shown that abnormal expression of miRNAs contributes to glioma growth [17]. However, the relationships between miRNAs and glioblastoma invasion need further investigation.

The aim of this study was to identify the role and underlying mechanism of NTSR1 in glioblastoma invasion. We found that NTSR1 knockdown suppressed glioblastoma invasion in vitro and in vivo. NTSR1 stimulated miR-494 transcription by promoting transcription factor Jun expression, and miR-494 was critical for NTSR1-induced glioblastoma invasion. Further investigation revealed that SOCS6 was a direct target of miR-494, and NTSR1 attenuated SOCS6 expression by upregulating miR-494 expression. SOCS6 was involved in NTSR1-induced glioblastoma invasion. This is a novel mechanism that could be exploited to develop a new therapeutic strategy for human glioblastoma.

\section{Materials and Methods}

\section{Cell culture}

Human glioblastoma cell lines (U87MG and U118MG) were purchased from ATCC. Cells were maintained in DMEM, 10\% FBS, and 1\% penicillin/streptomycin at 37 degrees centigrade and 5\% CO2 in water jacketed humidity controlled incubators. 


\section{Cellular Physiology Cell Physiol Biochem 2018;49:2382-2395

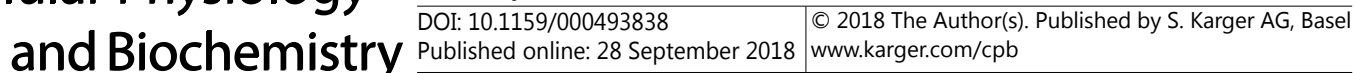 \\ Ou-Yang et al.: NTSR1 Promotes Glioblastoma Invasion}

\section{Reagents and Antibodies}

Chemical reagents were purchased from Sigma-Aldrich (ST. Louis, MO). Cell culture media and other supplements were purchased from Gibco Life Technologies (Carlsbad, CA, USA). NTSR1 antibody, Jun antibody and SOCS6 antibody (Abcam, Cambridge, UK). Tubulin antibody, GAPDH antibody and $\beta$-actin antibody (Santa Cruz, CA, USA).

\section{Plasmids, siRNAs, shRNAs, and Transfection}

Control sc-siRNA and siNTSR1 were purchased from Ambion. Control sc-shRNA and shNTSR1 were purchased from GENECHEM. Control sc-siRNA, siJun, and siSOCS6 were purchased from RIBOBIO. Human Jun cDNA was purchased from OriGene and then inserted into a pCMV6-XL4 vector (OriGene). Details are described in Supplementary Materials (for all supplementary material see www.karger. com/10.1159/000493838/).

\section{MiRNAs, anta-miRNAs, and Transfection}

Control sc-miRNA, miR-494 mimic, control anta-sc-miR and anta-miR-494 were purchased from RIBOBIO. U87MG and U118MG were cultured at a density of $1 \times 10^{6}$ cells in 60 -mm dishes in DMEM medium supplemented with $10 \%$ FBS. At $90 \%$ confluence, cells were transiently transfected with miRNAs or antamiRNAs using transfection reagent.

\section{Western blotting}

Cells were lysed in ice-cold RIPA buffer, and protein concentration was determined by a BCA protein assay kit (Santa Cruz, CA, USA). Protein mixed with sample buffer, boiled and subjected to SDS-PAGE and electroblotted onto PVDF membranes. Membranes were immunoblotted with the antibodies described in each experiment.

\section{Wound-healing and Transwell assays}

Cells were plated in 6-well plates, and wounds were made with a pipette tip after cells were attached. Images of the wound closure were photographed at $0 \mathrm{~h}$ and $24 \mathrm{~h}$. For invasion assay, transwell assay was performed with the $8 \mu \mathrm{m}$-pore chamber inserted into 24-well plates (Corning, NY, USA). Details are described in Supplementary Materials.

\section{Quantitative real-time PCR ( $q R T$-PCR)}

Total RNA from cells was extracted using the RNeasy mini kit (Qiagen). qRT-PCR of miR-494 expression was carried out using a TaqMan microRNA assay kit according to the manufacturer's protocol, U6 was used as an internal control. The primers of miR-494 were purchased from RIBOBIO.

\section{Chromatin immunoprecipitation (ChIP)}

ChIP was performed using the ChIP assay kit (Upstate Biotechnology). The binding region was spanning the Jun binding site (18.6kb upstream of pre-miR-494), a 31kb upstream of the pre-miR-494 5' end was used as a control (Control region) [18]. Details are described in Supplementary Materials

\section{Dual-Luciferase}

The pmiR-RB-Report ${ }^{\mathrm{TM}}$ luciferase miRNA expression reporter vector (RiboBio) was applied to construct the wild-type (WT) reporter vectors of the SOCS6 3' untranslated region (3' UTR). The coding sequence of miR-494 binding site was mutated to construct the mutant (MT) vector by site-directed mutagenesis. Details are described in Supplementary Materials

\section{Tumorigenesis studies}

Procedures involving mice were approved by the Institutional Animal Care and Use Committee of Army Medical University. The orthotopic xenografts model was established by injection of $1 \times 10^{7} \mathrm{sc}$-shRNAU87MG, shNTSR1-U87MG or shNTSR1+miR-494-U87MG cells into the right striatum of nude mice brains, respectively. MRI (Broker Biospec 7.0 Tesla Imaging System) was performed to detect tumor dimensions at 21th day after implantation, tumor volume $(\mathrm{Tv})=$ Length $\times \mathrm{Width}^{2} \times 0.52$. 


\section{Cellular Physiology Cell Physiol Biochem 2018;49:2382-2395 \begin{tabular}{l|l|l} 
and Biochemistry Published onlIne: 28 September 2018 & $\begin{array}{l}\text { (c) } 2018 \text { The Author(s). Published by S. Karger AG, Basel } \\
\text { www.karger.com/cpb }\end{array}$ \\
\hline
\end{tabular}

\section{Immunohistochemistry (IHC)}

The IHC staining of SOCS6 was performed using CECTASTAIN ABC Detection system (VECTOR). The result was determined according to the percentage of positive cell number to total cell number in 10 randomly selected microscopic fields.

\section{Statistical Analysis}

Statistical analyses were carried out using SPSS 13.0 statistical software. Statistical significance was calculated by Student's t-test or one-way ANOVA. Survival was analyzed by the Kaplan-Meier method and compared by log-rank test by GraphPad Prism 5. $\left(^{*}\right)$ p $<0.05$ were considered statistically significant.

\section{Results}

\section{NTSR1 Knockdown Suppressed Cell Migration and Invasion of Glioblastoma}

In an effort to investigate the role of NTSR1 in the migration and invasion of glioblastoma cells, we first silenced NTSR1 with a short interfering RNA (siNTSR1) in U87MG and U118MG cells (Fig. 1A). Next, we performed wound-healing assay to assess the effect of NTSR1 knockdown on cellular migratory activity within $24 \mathrm{~h}$. Transfection with siNTSR1 suppressed the migratory activity of U87MG and U118MG cells compared with control groups (Fig. 1B). However, siNTSR1 had no significant effects on glioblastoma cell proliferation within $24 \mathrm{~h}$ (Fig. S1A). Furthermore, The transwell assay showed that siNTSR1 transfection markedly attenuated the invasive activity of U87MG and U118MG cells (Fig. 1C). Taken together, the above data demonstrate that NTSR1 knockdown efficiently suppresses cellular migration and invasion of glioblastoma in vitro.

\section{NTSR1 Positively Regulated miR-494 Expression by Transcription Factor Jun}

To investigate the underlying mechanism by which NTSR1 enhances cell invasion in glioblastoma, we focused on miRNAs, which are a class of short noncoding RNAs that function as posttranscriptional regulators of gene expression [19]. Our previous work revealed that the expression of miR-494 is significantly downregulated after NTSR1 knockdown in U87MG cells [12]. Importantly, some studies have shown that miR-494 is critical for the invasion of glioblastoma cells $[20,21]$. To confirm the effect of NTSR1 knockdown on miR-494 expression, we measured miR-494 levels after siNTSR1 transfection in U87MG and U118MG cells. Indeed, we found that NTSR1 knockdown suppressed miR-494 expression in U87MG and U118MG cells (Fig. 2A). It has been reported that the upstream sequence of miR-494 promoter contains a Jun binding site (18.61kb upstream of the 5' end of pre-miR-494) [18]. To elucidate the direct involvement of transcription factor Jun in NTSR1-induced miR-494 expression, we first analyzed the effect of NTSR1 knockdown on Jun expression. We found that siNTSR1 suppressed the expression of Jun in U87MG and U118MG cells (Fig. 2B). Next, we carried out ChIP assay to verify the direct binding of Jun on miR-494 promoter in U87MG cells. As expected, ChIP analysis with a primer set covering the Jun binding site confirmed the physical association of Jun with miR-494 promoter by using an Jun antibody. In contrast, no chromatin enrichment by Jun antibody was observed in the control region $31 \mathrm{~kb}$ upstream of the 5' end of pre-miR-494, where there was no predicted binding site for Jun) (Fig. 2C).

To assess whether Jun is responsible for the transcriptional activation of miR-494 in glioblastoma cells, we silenced and overexpressed Jun in U87MG and U118MG cells with siJun and Jun vector, respectively (Fig. 2D and 2E). Jun knockdown significantly suppressed the expression of miR-494 in U87MG and U118MG cells (Fig. 2F). However, Jun overexpression promoted the expression of miR-494 in U87MG and U118MG cells (Fig. 2G). Taken together, these results suggest that transcription factor Jun, positively regulated by NTSR1, binds to miR-494 promoter and promotes miR-494 transactivation in glioblastoma cells. 


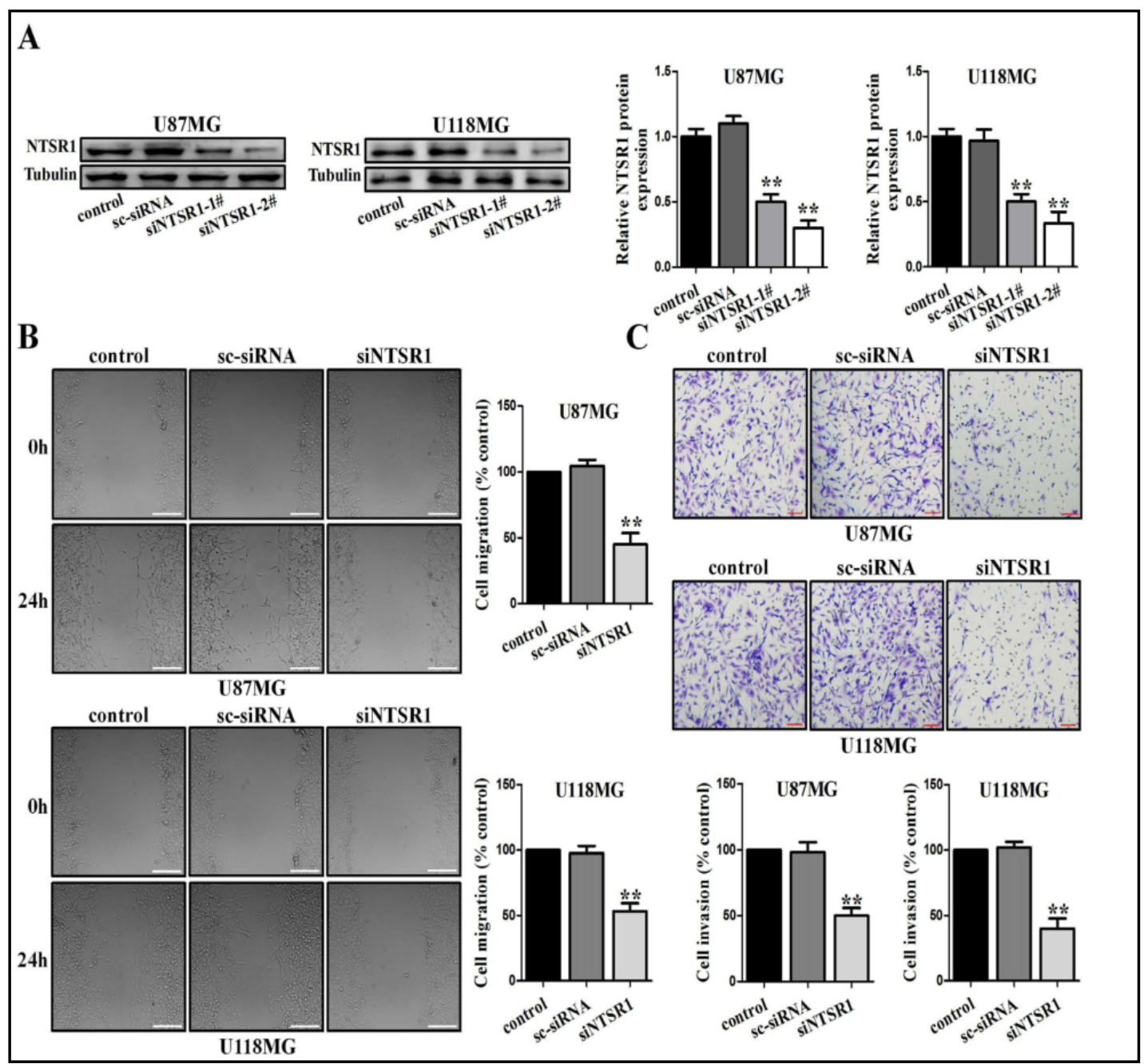

Fig. 1. NTSR1 knockdown suppressed glioblastoma cells migration and invasion. A. The protein levels of NTSR1 in the indicated cells were detected using western blotting assay. B. The wound-healing assay showed the migratory ability of U87MG and U118MG cells transfected with sc-siRNA or siNTSR1, the images of wound gap were taken for analysis. C. U87MG and U118MG cells were transfected with sc-siRNA or siNTSR1 for $24 \mathrm{~h}$. Invaded cells were stained and counted using microscopy. The histogram corresponds to the mean \pm S.D. of three independent experiments. ${ }^{* *} \mathrm{p}<0.01$.

MiR-494 Was Critical for NTSR1-induced Migration and Invasion of Glioblastoma Cells

To examine whether NTSR1-induced miR-494 plays a critical role in the invasive potential of glioblastoma cells, we first transfected miR-494 mimic into U87MG and U118MG cells. Quantitative real-time PCR showed that miR-494 levels were increased by at least 200 -fold compared with the control groups (Fig. S1B). We performed wound-healing assay to investigate the role of miR-494 in cell migration in glioblastoma, and found that miR494 significantly enhanced the cell migration of U87MG and U118MG cells with sc-siRNA transfection (Fig. 3A). MiR-494 also restored the migratory activity of U87MG and U118MG cells with siNTSR1 transfection (Fig. S1C). Transwell assay showed that miR-494 markedly enhanced glioblastoma cell invasion (Fig. 3B) and restored the invasive activity of siNTSR1transfected glioblastoma cells (Fig. S1D). Next, we used wound-healing assay to assess the role of miR-494 inhibitor (anta-miR-494) in cell migration in glioblastoma; we found that anta-miR-494 suppressed the cell migration of U87MG and U118MG cells with sc-siRNA transfection (Fig. 3C). Transwell assay showed that anta-miR-494 suppressed the invasive KARGER 


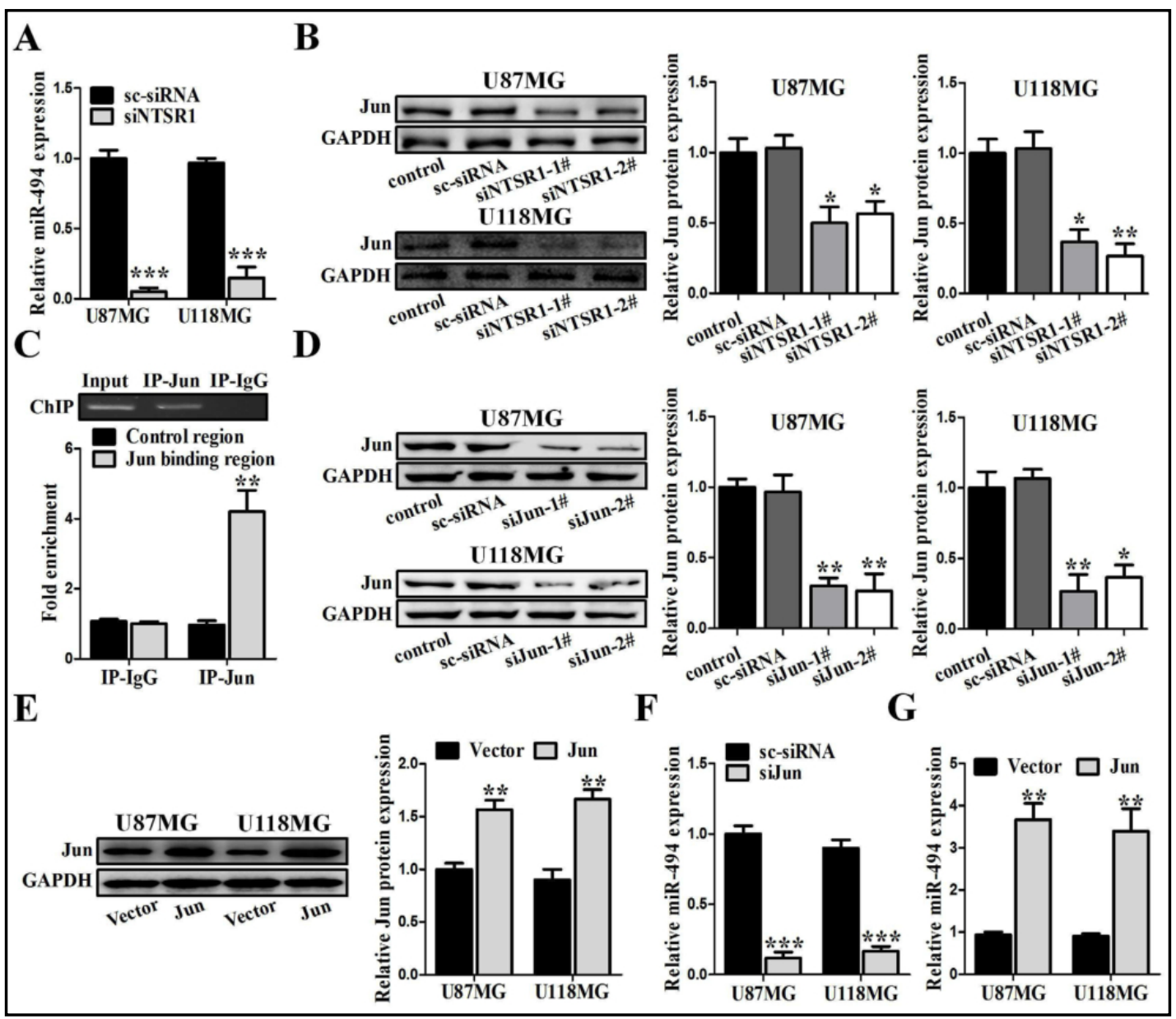

Fig. 2. Jun bound to the miR-494 promoter and promoted miR-494 expression. A. The levels of miR-494 were measured by qRT-PCR in the indicated U87MG and U118MG cells. B. The protein levels of Jun were detected using western blotting assay in the indicated U87MG and U118MG cells. C. ChIP demonstrated that precipitation with a Jun antibody led to an enrichment of miR-494 promoter fragment spanning Jun binding site compared with control groups. D-E. The protein levels of Jun were detected using western blotting assay in the indicated U87MG and U118MG cells. F-G. The levels of miR-494 were measured by qRT-PCR in the indicated U87MG and U118MG cells. The histogram corresponds to the mean \pm S.D. of three independent experiments. ${ }^{*} \mathrm{p}<0.05,{ }^{* *} \mathrm{p}<0.01,{ }^{* * *} \mathrm{p}<0.001$.

activity of U87MG and U118MG cells with sc-siRNA transfection (Fig. 3D). Taken together, the above data demonstrate that miR-494 is critical for NTSR1-induced migratory and invasive activities of glioblastoma cells.

\section{MiR-494 Directly Targeted SOCS6 3'UTR in Glioblastoma Cells}

We performed publicly available databases (www.targetscan.org, www.pictar.org, and www.microrna.org) to search the putative mRNA targets of miR-494 [22]. Among the candidate targets, we selected 3' UTR of SOCS6, which comprising three regions that matched the seed sequences of miR-494 (Fig. 4A). To verify whether SOCS6 is a direct target of miR494, a wild-type SOCS6 3' UTR containing the miR-494 binding site (WT) and a mutant 3' UTR (MT) were amplified and inserted into a dual-luciferase reporter, respectively. The luciferase activity assay showed that miR-494 significantly suppressed luciferase activity in the WT but not the MT reporter (Fig. 4B). To determine if miR-494 could affect SOCS6 protein expression in glioblastoma cells, we first transfected miR-494 mimic into U87MG and U118MG cells with sc-miRNA transfection. We found that siNTSR1 elevated the expression 


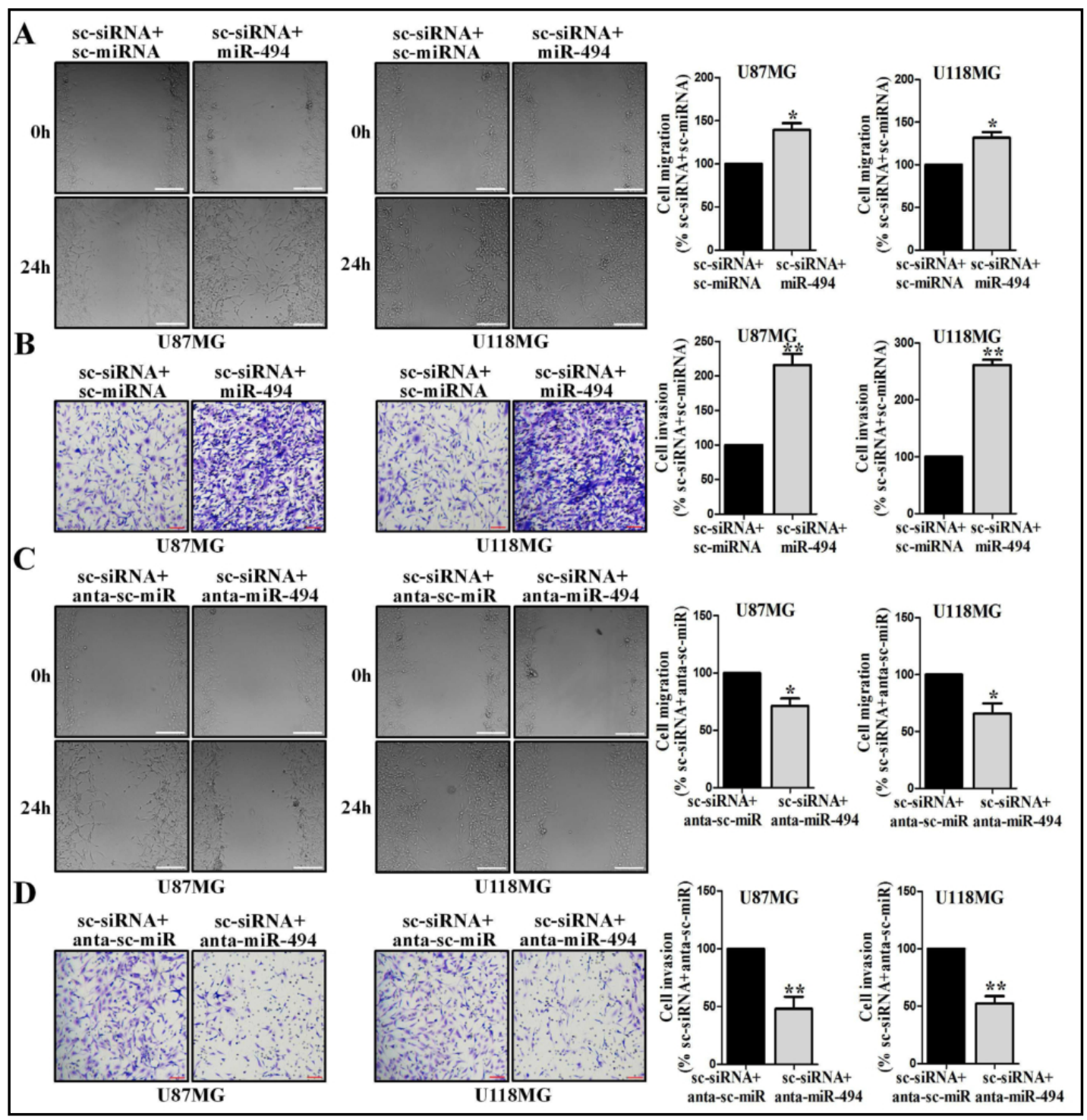

Fig. 3. miR-494 enhanced the migratory and invasive activities of glioblastoma cells. A. The woundhealing assay showed that miR-494 enhanced the migratory ability of U87MG and U118MG cells with scsiRNA transfection. B. The transwell assay showed that miR-494 enhanced the invasive ability of U87MG and U118MG cells with sc-siRNA transfection. C. The wound-healing assay showed that anta-miR-494 suppressed the migratory ability of U87MG and U118MG cells with sc-siRNA transfection. D. The transwell assay showed that anta-miR-494 attenuated the invasive ability of U87MG and U118MG cells with sc-siRNA transfection. The histogram corresponds to the mean \pm S.D. of three independent experiments. ${ }^{*} \mathrm{p}<0.05$, ${ }^{* *} \mathrm{p}<0.01$.

of SOCS6 in U87MG and U118MG cells with sc-miRNA transfection, and miR-494 suppressed the expression of SOCS6 in both sc-siRNA and siNTSR1 backgrounds (Fig. 4C). In addition, we also found that anta-miR-494 restored SOCS6 protein levels in U87MG and U118MG cells with sc-siRNA transfection; but, anta-miR-494 had no significant effects on SOCS6 protein levels in U87MG and U118MG cells with siNTSR1 transfection (Fig. 4D). Taken together, these results indicate that SOCS6 is a direct target of miR-494 in glioblastoma. 


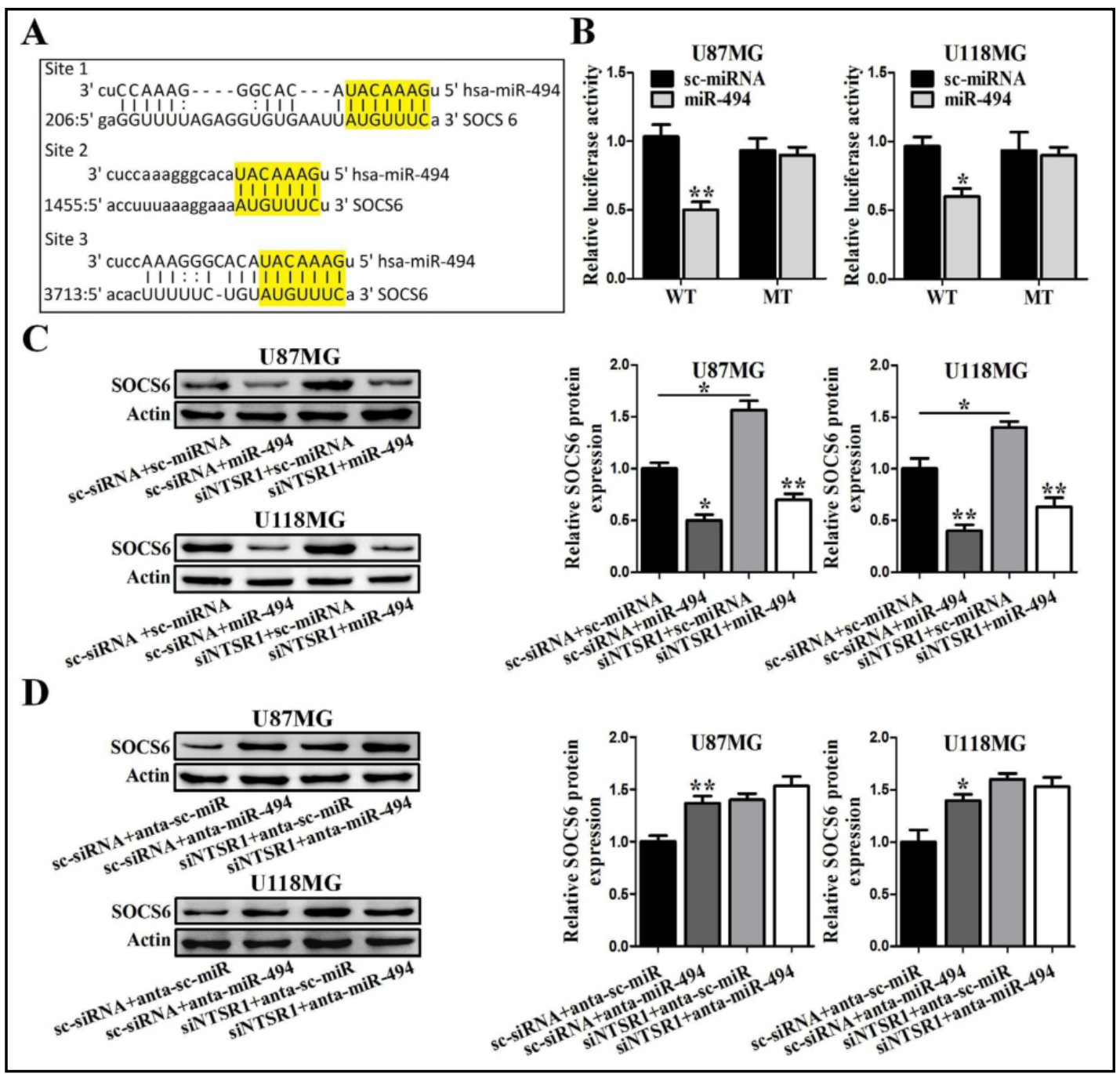

Fig. 4. miR-494 bound to SOCS6 3' UTR and negatively regulated SOCS6 expression. A. Predicted miR-494 target sequence in the $3^{\prime}$ UTR of SOCS6 gene. B. The reporter plasmids containing the wild-type 3' UTR of SOCS6 (WT) or mutant (MT) were co-transfected into U87MG and U118MG cells with miR-494 mimic or control sc-miRNA mimic to evaluate luciferase activity. C-D. The protein levels of SOCS6 were detected using western blotting assay in the indicated U87MG and U118MG cells. The histogram corresponds to the mean \pm S.D. of three independent experiments. ${ }^{*} \mathrm{p}<0.05,{ }^{* *} \mathrm{p}<0.01$.

\section{SOCS6 Was Involved in NTSR1-Promoted Glioblastoma Invasion}

We noted the important role of SOCS6 in solid tumor invasion [23, 24]. To investigate whether SOCS6 is essential for NTSR1-promoted glioblastoma cell migration and invasion, we first silenced SOCS6 in U87MG and U118MG cells (Fig. 5A). Next, we performed woundhealing assay and found that SOCS6 knockdown promoted the migratory activity of U87MG and U118MG cells with sc-siRNA transfection (Fig. 5B). In addition, SOCS6 knockdown also restored the migratory activity of U87MG and U118MG cells with siNTSR1 transfection (Fig. S2A). Finally, we found that SOCS6 knockdown increased the total number of invading cells in U87MG and U118MG cells with sc-siRNA transfection (Fig. 5C). SOCS6 knockdown also restored the invasive activity of U87MG and U118MG cells with siNTSR1 transfection (Fig. S2B). These results suggest that the promotional effect of NTSR1 on glioblastoma cells motility is dependent on SOCS6. 


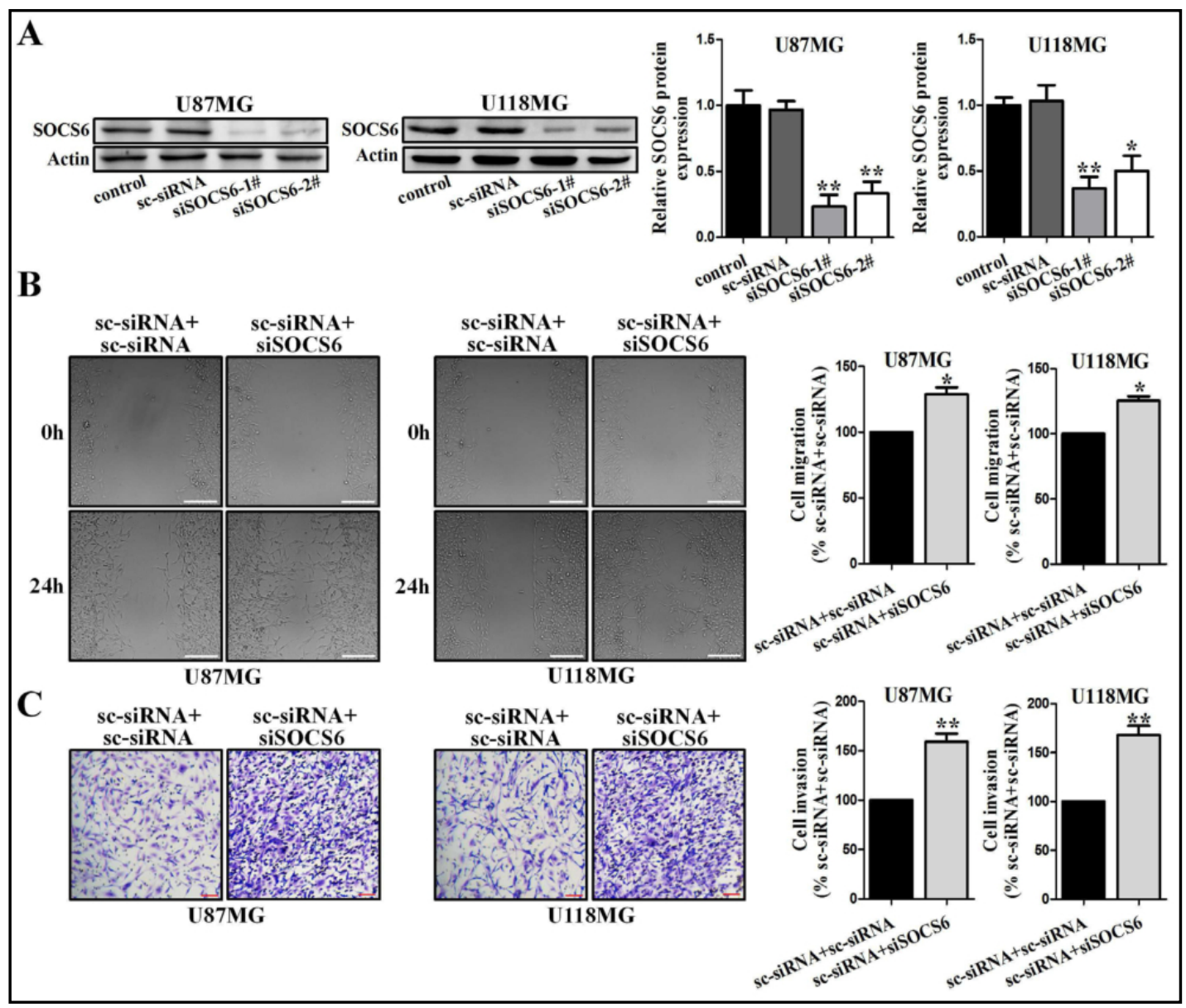

Fig. 5. SOCS6 knockdown enhanced the migratory and invasive activities of glioblastoma cells. A. The protein levels of SOCS6 were detected using western blotting assay in the indicated U87MG and U118MG cells. B. The wound-healing assay showed that SOCS6 knockdown enhanced the migratory ability of U87MG and U118MG cells with sc-siRNA transfection. C. The transwell assay showed that SOCS6 knockdown enhanced the invasive ability of U87MG and U118MG cells with sc-siRNA transfection. The histogram corresponds to the mean \pm S.D. of three independent experiments. ${ }^{*} \mathrm{p}<0.05,{ }^{* *} \mathrm{p}<0.01$.

\section{NTSR1 Knockdown Impaired Glioblastoma Invasion in vivo Through MiR-494}

To confirm the effect of NTSR1 knockdown and miR-494 upregulation on glioblastoma invasion in vivo, we first established shNTSR1-U87MG cells (Fig. 6A). Next, $1 \times 10^{7}$ of scshRNA-U87MG, shNTSR1-U87MG, and shNTSR1+miR-494-U87MG cells, respectively, were injected orthotopically into mice brains. We performed magnetic resonance imaging (MRI) to determine the tumor size at 21 days after the injection. Significant differences in the average volumes of the xenografts were seen between the sc-shRNA-U87MG and shNTSR1U87MG group. We also found that the average volumes of the xenografts were larger in the shNTSR1+miR-494-U87MG group compared with the shNTSR1-U87MG group (Fig. 6B and 6C). Immunohistochemical (IHC) staining was performed to detect the expression levels of SOCS6 in orthotopic glioblastoma. NTSR1 knockdown caused an increase in SOCS6 protein levels compared with the sc-shRNA-U87MG and shNTSR1+miR-494-U87MG groups (Fig. 6B). Hematoxylin-Eosin (H\&E) staining was performed to detect the invasiveness of the orthotopic glioblastoma. The sc-shRNA-U87MG group grew as extensively diffuse tumor cell clusters invading deep into the brain parenchyma. In contrast, NTSR1 knockdown produced tightly packed glioblastoma with relatively smooth borders. In addition, the shNTSR1+miR494-U87MG generated orthotopic glioblastoma was more aggressive than that of the 


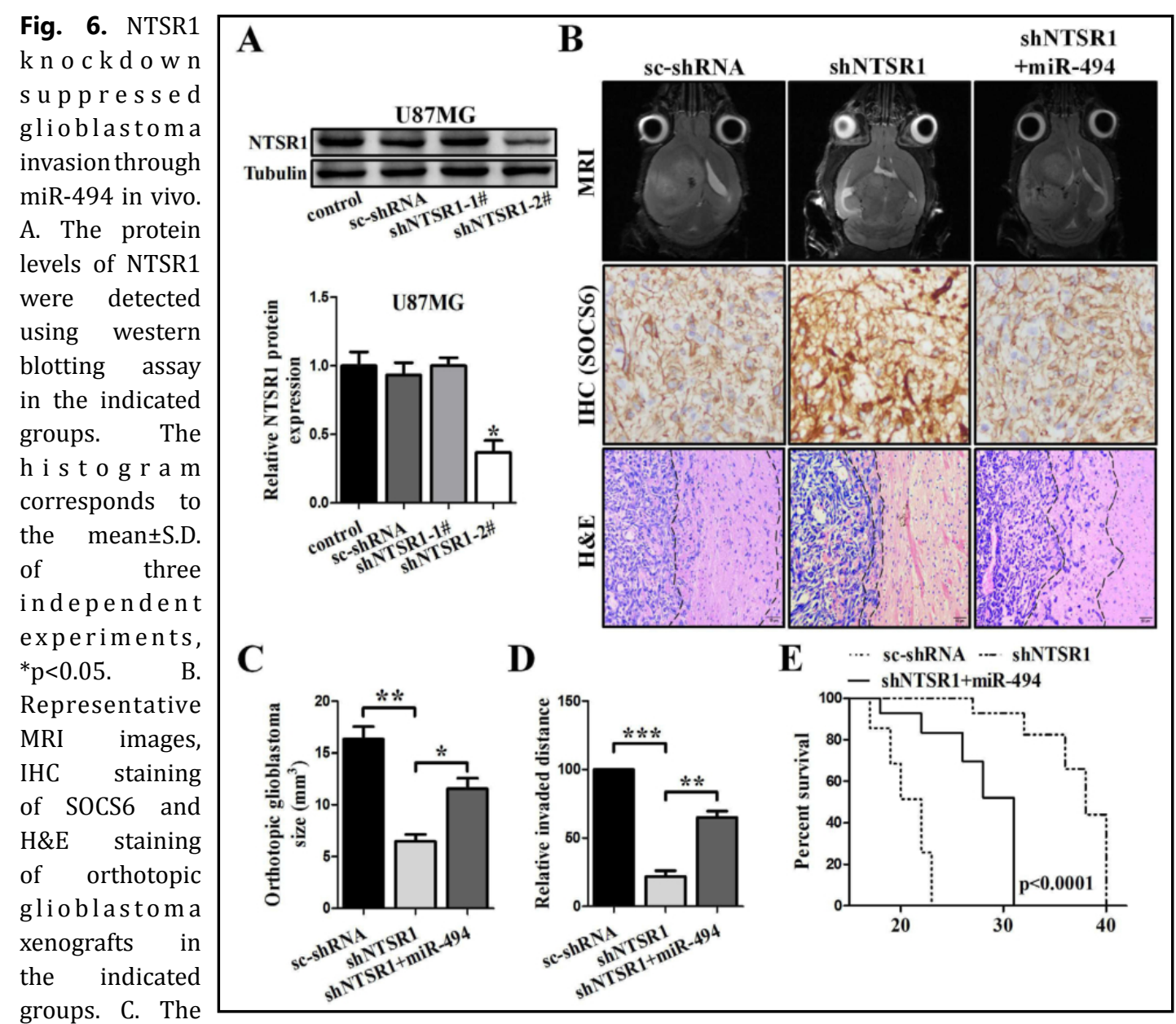

groups. C. The

average volumes

of the xenografts were measured at 21th day after implantation. D. Quantification of the average distance of orthotopic glioblastoma xenografts invasion in the indicated groups. E. The survival duration of tumorbearing mice was monitored in the indicated groups. The survival time was analyzed by the Kaplan-Meier method and compared by log-rank test. The histogram corresponds to the mean \pm S.D. of five independent experiments, ${ }^{*} \mathrm{p}<0.05,{ }^{* *} \mathrm{p}<0.01$ and ${ }^{* * *} \mathrm{p}<0.001$.

shNTSR1-U87MG group (Fig. 6B and 6D). Finally, we detected the effect of NTSR1 knockdown and miR-494 upregulation on the overall survival time of tumor-bearing mice. We found that NTSR1 knockdown alone prolonged the overall survival time of tumor-bearing mice, while miR-494 upregulation attenuated this survival advantage. These data indicate that NTSR1 knockdown impaired glioblastoma invasion and tumor growth through miR-494 in vivo.

\section{Discussion}

Aberrant expression of NTSR1 frequently occurs in human glioma, which plays important roles in various processes related to cancer biologies, such as cell proliferation, apoptosis, and the maintenance of glioma cell stemness [11-14]. In this study, we found that NTSR1 knockdown suppressed migration and invasion of glioblastoma cells in vitro and in vivo. More importantly, we identified miR-494 as an NTSR1-inducible miRNA that promoted cell migration and invasion, and was positively regulated by transcription factor Jun in U87MG and U118MG cells. In addition, we confirmed that the tumor suppressor SOCS6 was a direct 
target of miR-494, and was critical for NTSR1-induced glioblastoma invasion. Taken together, our present study demonstrated that NTSR1 promoted tumor migration and invasion via the Jun/miR-494/SOCS6 pathway in human glioblastoma (Fig. S3).

The highly invasive nature of glioblastoma is the main reason for recurrence after tumor resection [25]. The finding of the presence of NTSR1 in human glioblastoma facilitates our understanding of the mechanism underlying glioblastoma invasion. Our previous work demonstrated that activation of NTSR1 signaling is closely related to the poor prognosis of glioma patients, which enhances the proliferative and invasive characteristics of U87 and GL261 cell lines, while NTSR1 inhibition results in a decrease in cell proliferation and invasion; however, the underlying mechanism needs further investigation [11]. In our present study, we found that NTSR1 knockdown inhibited migration and invasion of U87MG and U118MG cells via the miR-494/SOCS6 axis in vitro and in vivo. It has been demonstrated that silencing NTSR1 alters a variety of miRNAs that have tumor-promoting and tumorsuppressing functions in glioblastoma cells $[12,14]$. These miRNAs may suppress the expression of target genes by specifically interacting with the $3^{\prime}$ UTR, and has a key role in the regulation of certain oncogenes and tumor suppressor genes in glioblastoma. In the present study, we identified that NTSR1 positively regulated miR-494 expression, while siNTSR1 significantly suppressed miR-494 expression in glioblastoma cells. However, the underlying mechanism of NTSR1 promotion of miR-494 expression is unclear. It has been established that the miR-494 promoter contains binding sites for Jun [18], which provides important clues for understanding the mechanism of miR-494 expression regulated by NTSR1. Our study demonstrated, using ChIP assay, that miR-494 was a direct transcriptional target of Jun. The increased or decreased miR-494 expression associated with Jun overexpression or downregulation also showed the transcriptional activation of miR-494 by Jun in glioblastoma. Jun is the important downstream target of MAPK family members ERK and JNK [26]. We previously established that NTSR1 signaling regulates activation of the ERK pathway in glioma [11]. In the present study, we revealed that NTSR1 signaling promoted miR-494 expression by upregulating Jun expression in glioblastoma cells.

In this study, we identified miR-494 as a siNTSR1-inducible miRNA that promoted glioblastoma invasion. Several reports on the invasion-promoting effect of miR-494 on cancer cells have been published. MiR-494 promotes cell migration and invasion by targeting PTEN in hepatocellular carcinoma and colorectal cancer $[27,28]$. MiR-494 enhances cellular proliferation and invasion by PTEN/AKT signaling in human glioblastoma cells [20]. These studies demonstrate the oncogenic characteristics of miR-494, while contrasting reports show that miR-494 is a tumor suppressor. In pancreatic cancer, MiR-494 exhibits cell viability and migration-suppressing effects via targeting c-myc, and SIRT1 [29]. Ectopic expression of miR-494 suppresses the metastatic potential of breast cancer by targeting PAK1 and CXCR4 $[30,31]$. Overexpression of miR-494 inhibits ovarian cancer cell migration and invasion by directly suppressing Cullin 4A expression [32]. MiR-494 inhibits invasion and proliferation of osteosarcoma by targeting insulin receptor substrate-1 [33]. In chondrosarcoma cells, miR-494 inhibits cell invasion by directly targeting SOX9 [34]. In prostate cancer, miR-494 targets CXCR4 to suppress invasion and migration [35]. The above studies concertedly imply that miR-494 has ambivalent functions in different cancers. In our study, NTSR1 positively regulated miR-494 expression in U87MG and U118MG cells, and we confirmed that miR-494 was critical for NTSR1-induced glioblastoma invasion. Therefore, miR-494 is an attractive candidate target to control glioblastoma invasion.

Human SOCS6 is a member of the SOCS family, and is located at locus 18q22.2 [36]. The SOCS family comprises 8 proteins, SOCS1 to SOCS7 and cytokine-inducible SH2-containing protein, which are characterized by an amino-terminal region of variable length, a central SH2 domain, and a carboxyl-terminal SOCS box [37]. Tumor suppressor function is proposed for SOCS6, and downregulation of SOCS6 has been observed in colorectal carcinoma and may serve as diagnostic biomarkers for colorectal carcinoma patients [38]. Some reports demonstrate that SOCS6 is critical for cancer invasion [24, 39]. In the present study, we found that SOCS6 knockdown enhanced glioblastoma cell migration and invasion, suggesting that 


\section{Cellular Physiology Cell Physiol Biochem 2018;49:2382-2395

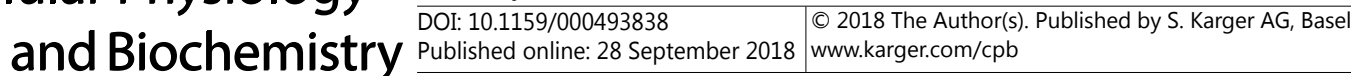 \\ Ou-Yang et al.: NTSR1 Promotes Glioblastoma Invasion}

SOCS6 is critical for glioblastoma invasion. It is now well established that SOCS6 regulates downstream signaling of several receptor tyrosine kinases including INSR, KIT, and FLT3, and these receptor tyrosine kinases are critical for cancer invasion [36, 40, 41]. However, whether SOCS6 regulates glioblastoma invasion via these receptor tyrosine kinases needs further investigation. It is reported that SOCS6 is a functional target of miR-301a, suggesting that SOCS6 is regulated by miRNAs in cancer. Here, we found that miR-494 directly targeted SOCS6 and that miR-494 upregulation could decrease endogenous SOCS6 protein levels in human glioblastoma cell lines. Together, these results indicate that miR-494 suppresses SOCS6, and thus promotes glioblastoma invasion.

\section{Conclusion}

In summary, our findings showed that NTSR1 knockdown suppressed cell migration and invasion in U87MG and U118MG glioblastoma cells. We identified a novel mechanism by which siNTSR1 suppressed glioblastoma invasion through the miR-494/SOCS6 axis in glioblastoma. Our findings may help to develop a therapeutic strategy that combines shNTSR1 and miR-494 inhibitors to counteract the high growth potential and invasiveness of human glioblastoma.

\section{Acknowledgements}

The results published here are in whole or part based upon data generated by TargerScan, PicTar, and microRNA. The excellent support of Dr. Yan Tan and Maojun Liao shall be gratefully acknowledged.

\section{Disclosure Statement}

The authors declare that they have no competing financial or non-financial interests.

\section{References}

1 Louis DN, Ohgaki H, Wiestler OD, Cavenee WK, Burger PC, Jouvet A, Scheithauer BW, Kleihues P: The 2007 WHO classification of tumours of the central nervous system. Acta Neuropathol 2007;114:97-109.

-2 Stupp R, Mason WP, van den Bent MJ, Weller M, Fisher B, Taphoorn MJ, Belanger K, Brandes AA, Marosi C, Bogdahn U, Curschmann J, Janzer RC, Ludwin SK, Gorlia T, Allgeier A, Lacombe D, Cairncross JG, Eisenhauer E, Mirimanoff RO: Radiotherapy plus concomitant and adjuvant temozolomide for glioblastoma. N Engl J Med 2005;352:987-996.

-3 Wen PY, Kesari S: Malignant gliomas in adults. N Engl J Med 2008;359:492-507.

-4 Eckert M, Klumpp L, Huber SM: Cellular Effects of the Antiepileptic Drug Valproic Acid in Glioblastoma. Cell Physiol Biochem 2017;44:1591-1605.

$>5$ Catalogna G, Talarico C, Dattilo V, Gangemi V, Calabria F, D’Antona L, Schenone S, Musumeci F, Bianco C, Perrotti N, Amato R, Cascini GL: The SGK1 Kinase Inhibitor SI113 Sensitizes Theranostic Effects of the 64CuCl2 in Human Glioblastoma Multiforme Cells. Cell Physiol Biochem 2017;43:108-119.

-6 Li H, Yuan X, Yan D, Li D, Guan F, Dong Y, Wang H, Liu X, Yang B: Long Non-Coding RNA MALAT1 Decreases the Sensitivity of Resistant Glioblastoma Cell Lines to Temozolomide. Cell Physiol Biochem 2017;42:11921201.

7 Mangiola A, de Bonis P, Maira G, Balducci M, Sica G, Lama G, Lauriola L, Anile C: Invasive tumor cells and prognosis in a selected population of patients with glioblastoma multiforme. Cancer 2008;113:841-846. 


\section{Cellular Physiology Cell Physiol Biochem 2018;49:2382-2395

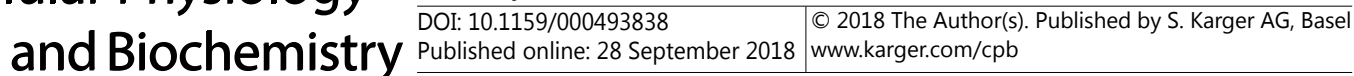

8 Tseliou M, Al-Qahtani A, Alarifi S, Alkahtani SH, Stournaras C, Sourvinos G: The Role of RhoA, RhoB and RhoC GTPases in Cell Morphology, Proliferation and Migration in Human Cytomegalovirus (HCMV) Infected Glioblastoma Cells. Cell Physiol Biochem 2016;38:94-109.

-9 Ouyang Q, Zhou J, Yang W, Cui H, Xu M, Yi L: Oncogenic role of neurotensin and neurotensin receptors in various cancers. Clin Exp Pharmacol Physiol 2017;44:841-846.

10 Xiao H, Zeng Y, Wang Q Wei S, Zhu X: A Novel Positive Feedback Loop Between NTSR1 and Wnt/betaCatenin Contributes to Tumor Growth of Glioblastoma. Cell Physiol Biochem 2017;43:2133-2142.

11 Ouyang Q, Gong X, Xiao H, Zhou J, Xu M, Dai Y, Xu L, Feng H, Cui H, Yi L: Neurotensin promotes the progression of malignant glioma through NTSR1 and impacts the prognosis of glioma patients. Mol Cancer 2015;14:21.

12 Ouyang Q, Chen G, Zhou J, Li L, Dong Z, Yang R, Xu L, Cui H, Xu M, Yi L: Neurotensin signaling stimulates glioblastoma cell proliferation by upregulating c-Myc and inhibiting miR-29b-1 and miR-129-3p. Neuro Oncol 2016;18:216-226.

13 Zhou J, Yi L, Ouyang Q Xu L, Cui H, Xu M: Neurotensin signaling regulates stem-like traits of glioblastoma stem cells through activation of IL-8/CXCR1/STAT3 pathway. Cell Signal 2014;26:2896-2902.

14 Dong Z, Lei Q, Yang R, Zhu S, Ke XX, Yang L, Cui H, Yi L: Inhibition of neurotensin receptor 1 induces intrinsic apoptosis via let-7a-3p/Bcl-w axis in glioblastoma. Br J Cancer 2017;116:1572-1584.

15 Fabian MR, Sonenberg N, Filipowicz W: Regulation of mRNA translation and stability by microRNAs. Annu Rev Biochem 2010;79:351-379.

16 Peng Y, Croce CM: The role of MicroRNAs in human cancer. Signal Transduct Target Ther 2016;1:15004.

17 Ouyang Q, Xu L, Cui H, Xu M, Yi L: MicroRNAs and cell cycle of malignant glioma. Int J Neurosci 2016;126:19.

-18 Romano G, Acunzo M, Garofalo M, Di Leva G, Cascione L, Zanca C, Bolon B, Condorelli G, Croce CM: MiR-494 is regulated by ERK1/2 and modulates TRAIL-induced apoptosis in non-small-cell lung cancer through BIM down-regulation. Proc Natl Acad Sci U S A 2012;109:16570-16575.

19 Cheng CJ, Bahal R, Babar IA, Pincus Z, Barrera F, Liu C, Svoronos A, Braddock DT, Glazer PM, Engelman DM, Saltzman WM, Slack FJ: MicroRNA silencing for cancer therapy targeted to the tumour microenvironment. Nature 2015;518:107-110.

20 Li XT, Wang HZ, Wu ZW, Yang TQ, Zhao ZH, Chen GL, Xie XS, Li B, Wei YX, Huang YL, Zhou YX, Du ZW: miR494-3p Regulates Cellular Proliferation, Invasion, Migration, and Apoptosis by PTEN/AKT Signaling in Human Glioblastoma Cells. Cell Mol Neurobiol 2015;35:679-687.

-21 Kwak SY, Yang JS, Kim BY, Bae IH, Han YH: Ionizing radiation-inducible miR-494 promotes glioma cell invasion through EGFR stabilization by targeting p190B rhoGAP. Biochim Biophys Acta 2014;1843:508516.

-22 Roberts JT, Borchert GM: Computational Prediction of MicroRNA Target Genes, Target Prediction Databases, and Web Resources. Methods Mol Biol 2017;1617:109-122.

23 Kumar S, Lu B, Dixit U, Hossain S, Liu Y, Li J, Hornbeck P, Zheng W, Sowalsky AG, Kotula L, Birge RB: Reciprocal regulation of Abl kinase by Crk Y251 and Abi1 controls invasive phenotypes in glioblastoma. Oncotarget 2015;6:37792-37807.

-24 Fang Y, Sun B, Xiang J, Chen Z: MiR-301a promotes colorectal cancer cell growth and invasion by directly targeting SOCS6. Cell Physiol Biochem 2015;35:227-236.

25 Mao L, Whitehead CA, Paradiso L, Kaye AH, Morokoff AP, Luwor RB, Stylli SS: Enhancement of invadopodia activity in glioma cells by sublethal doses of irradiation and temozolomide. J Neurosurg 2017;1-13.

-26 Kim EK, Choi EJ: Compromised MAPK signaling in human diseases: an update. Arch Toxicol 2015;89:867882.

27 Liu K, Liu S, Zhang W, Jia B, Tan L, Jin Z, Liu Y: miR-494 promotes cell proliferation, migration and invasion, and increased sorafenib resistance in hepatocellular carcinoma by targeting PTEN. Oncol Rep 2015;34:1003-1010.

28 Sun HB, Chen X, Ji H, Wu T, Lu HW, Zhang Y, Li H, Li YM: miR494 is an independent prognostic factor and promotes cell migration and invasion in colorectal cancer by directly targeting PTEN. Int J Oncol 2014;45:2486-2494.

29 Liu Y, Li X, Zhu S, Zhang JG, Yang M, Qin Q, Deng SC, Wang B, Tian K, Liu L, Niu Y, Wang CY, Zhao G: Ectopic expression of miR-494 inhibited the proliferation, invasion and chemoresistance of pancreatic cancer by regulating SIRT1 and c-Myc. Gene Ther 2015;22:729-738. 


\section{Cellular Physiology Cell Physiol Biochem 2018;49:2382-2395 \begin{tabular}{ll|l} 
DoI: 10.1159/000493838 & 2018 The Author(s). Published by S. Karger AG, Basel \\
and Biochemistryer.com/cpb
\end{tabular}

30 Zhan MN, Yu XT, Tang J, Zhou CX, Wang CL, Yin QQ, Gong XF, He M, He JR, Chen GQ Zhao Q: MicroRNA-494 inhibits breast cancer progression by directly targeting PAK1. Cell Death Dis 2017;8:e2529.

-31 Song L, Liu D, Wang B, He J, Zhang S, Dai Z, Ma X, Wang X: miR-494 suppresses the progression of breast cancer in vitro by targeting CXCR4 through the Wnt/beta-catenin signaling pathway. Oncol Rep 2015;34:525-531.

-32 Han X, Fang Z, Wang H, Jiao R, Zhou J, Fang N: CUL4A functions as an oncogene in ovarian cancer and is directly regulated by miR-494. Biochem Biophys Res Commun 2016;480:675-681.

-33 Zhi X, Wu K, Yu D, Wang Y, Yu Y, Yan P, Lv G: MicroRNA-494 inhibits proliferation and metastasis of osteosarcoma through repressing insulin receptor substrate-1. Am J Transl Res 2016;8:3439-3447.

-34 Li J, Wang L, Liu Z, Zu C, Xing F, Yang P, Yang Y, Dang X, Wang K: MicroRNA-494 inhibits cell proliferation and invasion of chondrosarcoma cells in vivo and in vitro by directly targeting SOX9. Oncotarget 2015;6:2621626229.

-35 Shen PF, Chen XQ, Liao YC, Chen N, Zhou Q, Wei Q, Li X, Wang J, Zeng H: MicroRNA-494-3p targets CXCR4 to suppress the proliferation, invasion, and migration of prostate cancer. Prostate 2014;74:756-767.

-36 Kabir NN, Sun J, Ronnstrand L, Kazi JU: SOCS6 is a selective suppressor of receptor tyrosine kinase signaling. Tumour Biol 2014;35:10581-10589.

-37 Kazi JU, Kabir NN, Flores-Morales A, Ronnstrand L: SOCS proteins in regulation of receptor tyrosine kinase signaling. Cell Mol Life Sci 2014;71:3297-3310.

-38 Letellier E, Schmitz M, Baig K, Beaume N, Schwartz C, Frasquilho S, Antunes L, Marcon N, Nazarov PV, Vallar L, Even J, Haan S: Identification of SOCS2 and SOCS6 as biomarkers in human colorectal cancer. Br J Cancer 2014;111:726-735.

39 Li ZB, Li ZZ, Li L, Chu HT, Jia M: MiR-21 and miR-183 can simultaneously target SOCS6 and modulate growth and invasion of hepatocellular carcinoma (HCC) cells. Eur Rev Med Pharmacol Sci 2015;19:32083217.

40 Sun Z, Liu Q, Hong H, Zhang H, Zhang T: miR-19 promotes osteosarcoma progression by targeting SOCS6. Biochem Biophys Res Commun 2018;495:1363-1369.

-41 Kazi JU, Sun J, Phung B, Zadjali F, Flores-Morales A, Ronnstrand L: Suppressor of cytokine signaling 6 (SOCS6) negatively regulates Flt3 signal transduction through direct binding to phosphorylated tyrosines 591 and 919 of Flt3. J Biol Chem 2012;287:36509-36517. 